\title{
Las sociologías post contemporáneas: discusiones teóricas, estrategias metodológicas y prácticas de investigación en contextos diferentes
}

\author{
Post contemporary sociologies: theoretical discussions, \\ methodological strategies and research practices in different \\ contexts
}

\section{Gabriel Nardacchione * y Juan Ignacio Piovani **}

\begin{abstract}
* CONICET - Instituto de Investigaciones en Ciencias Sociales "Gino Germani" Facultad de Ciencias Sociales - Universidad de Buenos Aires, Argentina I gabriel.nardacchione@gmail.com

** Centro Interdisciplinario de Metodología de las Ciencias Sociales (CIMeCS) Facultad de Humanidades y Ciencias de la Educación Universidad Nacional de La Plata - CONICET, Argentina | juan.piovani@presi.unlp.edu.ar
\end{abstract}

\section{PALABRAS CLAVE}

Teoría social clásica Teoría social contemporánea Sociología post contemporánea

\section{KEYWORDS}

Classic social theory Contemporary social theory Post contemporary sociology

\section{RESUMEN}

En este artículo se presenta un breve análisis de las contribuciones teóricas y empíricas que forman parte del presente dossier, enmarcándolas en una definición de la sociología post contemporánea que se construye a partir de la consideración de cómo se ha tratado a las teorías clásicas y contemporáneas en la enseñanza de la sociología.

\begin{abstract}
In this article we present a brief analysis of the theoretical and empirical contributions included in this dossier, framing them within a definition of post contemporary sociology that stems from the consideration of how classic and contemporary theories have been treated in the scholarly programs of sociology.
\end{abstract}

Cita sugerida: Nardacchione, G. y Piovani, J. I. (2017). Las sociologías post contemporáneas: discusiones teóricas, estrategias metodológicas y prácticas de investigación en contextos diferentes. Cuestiones de Sociología, 16, e023. https://doi.org/10.24215/23468904e023 
La institucionalización de la sociología en el ámbito universitario ha implicado, entre otras cosas, la necesidad de contar con contenidos organizados y sistematizados que puedan transmitirse en el marco de programas de estudiodirigidos a formar las nuevas generaciones de sociólogos. Esto no significa desconocer que el pensamiento sociológico, como puede constarse en cualquier libro que aborde su historia (por ejemplo Aron 1967; Nisbet, 1969) antecede ampliamente este problema de la enseñanza formal, que recién comienza a tomar forma entre fines del siglo XIX y principios del siglo $\mathrm{XX}$, y que se estructura en torno de cómo organizar el conocimiento sociológico con fines didácticos, con las consiguientes formas de resolverlo, ya sea siguiendo un criterio histórico o sistemático, por autores, escuelas, temas/problemas, etc.En este sentido, resulta importante señalar la diferencia entre el conocimiento sociológico y la sociología como disciplina institucionalizada, para cuya reproducción adquiere relevancia la transmisión de un conocimiento sociológico que ha sido previamente seleccionado, organizado y sistematizado.

En esta organización de contenidos sociológicos “enseñables”, las cuestiones teóricas y metodológicas han tenido un lugar especialmente importante. En un artículo previo (Piovani, 2015) se ha abordado la forma en que fue constituyéndose un campo metodológico en relación con la formación de los sociólogos. Esto llevó, progresivamente,a la definición de ciertos contenidos canónicos acerca de los instrumentos conceptuales y operativos válidos para la construcción de conocimiento "científico-social” y de un corpus de obras especializadasque abordan dichos instrumentos, en general con el formato de manual.

En relación con la enseñanza de la teoría sociológica, o incluso de la sociología como disciplina científica en un sentido más amplio (con sus perspectivas teóricas y autores referentes), tal vez la obra de Park y Burgess, Introductionto the Science of Sociology (1921) —conocida como Green Bible - , constituya un primer ejemplo relevante, ya que no se trata del texto de uno u otro autor clave para la disciplina, sino de un conjunto de contenidos temáticos (y de autores) organizado y sistematizado a los efectos de la formación sociológica universitaria. El hecho de que fuera popularmente conocido como "biblia" deja entrever su importancia en esa época, al menos en el marco del Departamento de Sociología de Chicago, primero en constituirse a nivel mundial.

A igual que en el caso de la metodología, la progresiva consolidación de la enseñanza universitaria de la sociología también fue dando lugar a formas relativamente canónicasde organizar el tratamiento de los contenidos teóricos de la disciplina, ampliamente compartidas en diferentes contextos institucionales y nacionales. Al menos en América Latina la distinción entre “clásica” y “contemporánea” ha sido un recurso organizador clave para dichos contenidos, más allá de que ellos se aborden en el marco de cursos de "teoría”, de "(historia del) pensamiento sociológico” o de “sociologíasistemática” (aun reconociendo las importantes diferencias entre estas modalidades y estrategias).

Pero,¿cómo se ha definido a las teorías clásicas y contemporáneas? ¿Qué tradiciones, temas y autores han sido recurrentemente abordados en cada una deellas?Estas preguntas no tienen una respuesta unívoca y no es el objeto de este artículo entrar en los debates especializados o abordar con profundidad la cuestión. Pero al menos una aproximación general resulta indispensable para poder conferirle sentido a la expresión “sociologías post contemporáneas” que hemos adoptado como clave para la estructuración del presente dossier. 
Un buen punto de partida podría ser lo que los propios académicos de la sociología consideran como sus autores y obras centrales. En este sentido cobran relevancia los datos recabados por la Asociación Internacional de Sociología durante su Congreso Mundial de Montreal (1998), cuando se consultó a los asistentes sobre los textos más importantes de la disciplina. A partir del análisis de estos datos, y en el marco de una sociología de la sociología, Lamo de Espinosa, E. (2001) resume lahistoria de la sociologíaa partir de cinco generaciones de pensadores: (1) pioneros, (2) fundadores, (3) institucionalizadores, (4) compiladores y (5) constructivistas.Como se verá más adelante, este resumen incluye todos los autores y tradiciones que se consideran habitualmentepertinentes para definirlo clásicoy lo contemporáneo en el marco del tratamiento sistemático de la teoría sociológica y de su enseñanza.

Hernández Romero y Galindo Sosa (2009) señalan quepuede considerarse clásica la obra que funciona como modelo o ideal, o que resulta central dentro de un ámbito específico. Partiendo de esta definición, sostienen que "actualmente, existe consenso respecto a la centralidad de la propuesta teórico-metodológica desarrollada por Durkheim, Weber y Marx, lo que los ha colocado en el estatus de clásicos de la teoría sociológica”. Es por ello que en el proceso de formación académica del sociólogo, agregan, es posible encontrar en cualquier plan de estudios por lo menos un curso de teoría clásica, que implícita o explícitamente analice a estos tres pensadores.

La postura de estos autores en relación con la teoría clásica expresa lo que podríamos considerar un “consenso de mínima” 1 , en el sentido de que virtualmente no existen historiadores de la disciplina ni programas de formación en teoría sociológica que no reconozcan la centralidad de estos tres autores o que no analicen en detalle su obra. Sin embargo, es frecuente encontrar otras posturas que amplían el alcance temporal y/o autoral de la teoría clásica.

Gane (2005), por ejemplo, en su definición de sociología clásica toma a estos tres sociólogos pero agrega a Comte, habitualmente considerado el fundador de la disciplina. Y esta definición“apenas expandida” es bastante frecuente en los programas universitarios.

Por otra parte, como señala Ritzer (1993), “a medida que pasa el tiempo [otros]pensadores van entrando a formar parte de la tradición clásica”, y es así que en su conocido manual, titulado justamente Teoría Social Clásica, aun sin descuidar la centralidad de Marx, Durkheim y Weber - junto a quienes incluye a Simmel-, agrega a tres autores del siglo XX(Mead, Schutz y Parsons)ya dos sociólogos fundacionales (Comte y Spencer).

En una línea similar podría considerarse la propuesta de J. Alexander, para quien las obras clásicas remiten a un conjunto limitado de textos que todos conocemos y que permiten tener un lenguaje común (Zabludovsky, 1991). En sus 4 volúmenes sobre la lógica teórica en sociología, publicados entre 1982 y 1984, incluye primero el tratamiento del Positivismo sociológico (originado en Comte), luego la obra de Marx y Durkheim, a continuación la de Weber y finalmente la de Parsons.

Sin especificar autores o perspectivas puntuales, Bialakowsky (2010) sostiene que la sociología clásica es la “que recorre todo el siglo XIX hasta el fin de la Primera Guerra Mundial”. Este criterio cronológico permite incorporar a la definición de clásicos a autores como Saint Simon (aunque haya nacido en la segunda mitad del siglo XVIII), Töenniesy Pareto, quetambién suelenaparecer considerados como tales. 
En resumen, y volviendo a la propuesta de Lamo de Espinoza (2001), puede constatarse que las definiciones más habituales de teoría clásica incluyen a los más destacados exponentes de lo que define como "fundadores" (Saint Simon, Comte, Tocqueville, Marx y Spencer) e “institucionalizadores” (Durkheim, Pareto, Weber, Simmel, Mead y Töennies, junto con los clásicos de la Escuela de Chicago). La situación de los “compiladores” es un poco más ambigua, ya que algunos de sus exponentes han sido tratados como referentes de la sociología clásica o de la contemporánea (en definitiva, nada impide -y tal vez de aquí surja en parte la ambigüedadconsiderar clásico a un autor cronológicamente contemporáneo). Nos referimos tanto a los que Lamo de Espinoza considera compiladores de raigambre conservadora, como Parsons, o crítica, como Wright Mills,Adorno y Horkheimer, entre otros.

Dubet (1996), al igual que Ritzer (1993), ubica a Parsons entre los clásicos. Pero en la organización didáctica de la teoría sociológica, al menos en Argentina, este autor suele aparecer encuadrado entre los contemporáneos. Esto es así porque resultahabitualordenar los contenidos de las asignaturas que llevan el rótulo de "contemporáneas”a partir de lo que Giddens denominó “consenso ortodoxo", definido en torno de la sociología académica norteamericana y, más puntualmente, del estructuralfuncionalismo, con Parsons y Merton a la cabeza, por un lado, y del “disenso ortodoxo”, con énfasis en autores como Giddens, Bourdieu yHabermas, por el otro. Justamente estos últimos sociólogos son los que Lamo de Espinoza (2001) incluye entre los “constructivistas”, figuras centrales de la sociología de finales de siglo XX y, a su juicio, responsables de haber recuperado la preocupación por la Gran Teoría.

Si se revisa los programas actuales de asignaturas dedicadas a la teoría social contemporánea en Argentina podrá constatarse su consistencia con lo que se acaba de argumentar en el párrafo precedente. Pereyra y Serrani, por ejemplo, son responsables del dictado de un curso en la Universidad de San Martín que define a la teoría contemporánea a partir de la consolidación de las ciencias sociales con posterioridad a la Segunda Guerra Mundial. De esto modo, incluyen la perspectiva estructural-funcionalista (centrándose en Parsons y Merton), un conjunto variado de perspectivas críticas del funcionalismo (Teoría Crítica, Schutz, Goffman, Becker y Wright Mills), el disenso ortodoxo (Bourdieu, Giddens y Habermas), yalgunas perspectivas más actuales (Beck, Latour, Bauman).

En una línea en parte similar, Camou y equipo docente, responsables de la misma asignatura en la carrera de sociología de la Universidad de La Plata 2 , organizan los contenidos en torno de "cuatro grandes cauces por los que ha venido discurriendo el análisis sociológico”: la tradición sistémica (Parsons, Merton y otros), el modelo del orden conflictivo (Foucault, Bourdieu, Touraine, Habermas, Giddens y otros), el de la "elección racional” (entre ellos Olson, Elster, Boudon) y la tradición “microsociológica” (Goffman, Garfinkel, Berger, Luckmann).

Más allá de los matices que se puedan observar en las definiciones de "teoría contemporánea”, y aún reconociendo que ella comprende una diversidad de tradiciones y autores, existe cierto consenso en torno de la centralidad de lo que Lamo de Espinoza llama "constructivistas" y que otros encuadran en el “disenso ortodoxo": Bourdieu, Giddens, Habermas. Y entre ellos, al menos en Argentina (tal vez también en Brasil y otros países latinoamericanos), Bourdieu ha sido sin dudas la referencia más importante. 
La afirmación precedente puede sostenerse con argumentos cualitativos y cuantitativos. Desde un punto de vista cualitativo, podría decirse que dentro de una pretensión común por la Gran Teoría, la sociologíade Bourdieu (1970, 1980, 1988) atraviesa de manera consistente y con base empírica, por un lado, diversas áreas problemáticas, a saber: el estudio de laburocracia, del sistema educativo, de la producción cultural, de las prácticas mediáticas, entre otras.Por otra parte, puede dialogar con un gran número de disciplinas: en principio con la filosofía, pero sin duda con la antropología, con los estudios políticos, con las ciencias de la educación y de la administración, con los estudios del arte y de la comunicación. De manera análoga a la teoría parsoniana (aunque con supuestos radicalmente diferentes) suteoría de los campos le permite un transporte de su concepción a las distintas esferas de lo social. A nuestro criterio esto es logrado sólo parcialmente en la obra Giddens y con mucho menos base empírica en la obra de Habermas. Desde un punto de vista cuantitativo se constata, siguiendo el análisis de Baranger (2011) sobre la producción escrita de sociólogos (y antropólogos) argentinos a partir de sus presentaciones en 4 congresos nacionales o regionales celebrados entre 2008 y 2009 (IX Congreso Argentino de Antropología Social - CAAS; V Jornadas de Sociología de la UNLP - JSOC; I Encuentro Latinoamericano de Metodología de las Ciencias Sociales - ELMECS; XXVII Congreso de la Asociación Latinoamericana de Sociología - ALAS) que Bourdieu aparece sistemáticamente como el autor más citado en los 4 congresos, mientras que Giddens resulta quinto en ALAS, décimo en JSOC y decimonoveno en ELMECS, y Habermas aparece octavo en ALAS y decimosegundo en JSOC.

Más arriba se dijo tangencialmente que la distinción entre clásicos y contemporáneos (para referirse a los autores) puede ser en cierto sentido engañosa, porque en definitiva, la organización de los contenidos teóricos -a los efectos de su enseñanza- gira mayoritariamente en torno de lo que en un contexto determinado se consideran referencias clásicas, independientemente de su adscripción a uno $u$ otro de los dos bloques que se han analizadoaquí y que, como se ha señalado,suelendelimitarse siguiendocriterios vinculados con el desarrollo histórico de las disciplina. Se podría hablar entonces de clásicos del período clásico y clásicos contemporáneos. Y si bien las razones por las cuales un autor pasa a ser considerado“clásico”son complejas, sin dudas resulta relevante una cierta distancia temporal que permita la amplia recepción y "sedimentación” de su obra en la comunidad académica. Esto, a lo que probablemente también contribuya la limitación de tiempo para el desarrollo de contenidos dentro de los planes de estudio y los programas de asignaturas, suele impedir -o al menos reducir los espacios para- el tratamiento de las más actuales construcciones teóricas.

Sin embargo, es evidente que la elaboración y reflexión teóricas son dinámicas, porque se alimentan tanto de la recepción y crítica de autores y obras previas como de investigaciones empíricas sobre problemas y configuraciones sociales cambiantes, o emergentes, que demandan innovaciones conceptuales y explicativas. Es justamente en este espacio de las más actuales elaboraciones teóricas de la sociología que se sitúan las discusiones presentadas en este dossier, a las que hemos definido como “post contemporáneas” por dos razones: una de tipo cronológico, porque se produjeron temporalmente después de lo que canónicamente se ha establecido como "teoría sociológica contemporánea” (más allá de los matices que se han señaladoal respecto) y otra sustantiva, porque son discusiones en torno del legado de la obra del autor que podríamos considerar más destacado entre los clásicos contemporáneos: Pierre Bourdieu. 
En efecto, el dossier se centra en los actuales aportes pragmatistas al debate de la sociología de Bourdieu. Estos aportes los señalamos “en plural” dado el carácter relativamente marginal de los mismos en relación con la sociología canónica y dadas las fuertes controversias internas que caracterizaron al pragmatismo desde su origen como matriz de pensamiento. Su marginalidad se observa fundamentalmente en el marco de la sociología europea (continental) clásica e incluso en lasociología norteamericana ${ }^{\underline{3}}$, a pesar de su relativa influencia en esta última. Las controversias remiten a cierta imposibilidad del pragmatismo, desde sus inicios, de encontrar un tronco común con el cual identificarse ${ }^{4}$. De allí que a menudo haya sido unificado a través de nociones como "un estilo" o "una actitud”; fuertemente asociado a un abordaje metodológico más que a una consistencia teórica (Perez Tudela, 2008;Cometti, 2010).

El recorrido de este dossier apunta a diferentes contextos: Francia, Estados Unidos y América Latina. Nos interesa la relación de mutua influenciaentre EE.UU. y Francia, tanto en lo que hace a la lectura de Bourdieu en el marco de una tradición pragmática (en un sentido laxo) dentro de la sociología norteamericana como, en el caso simétrico,la manera en que la tradición pragmatistaestadounidensecomenzó a generar influencia en la sociología canónica franco-francesa (fuertemente reactiva al paradigma anglosajón) desde la última década del siglo XX (Bartheet al, 2016; Breviglier yStavoDebauge, 1999).Peroen particular nos interesan las repercusiones que estos debates pueden tener en América latina, y esto es por dos motivos. Primero por su oportunidad, considerando un cierto agotamiento de los modelos teóricos y epistemológicos (“contemporáneos”) y frente a una inclinación relativamente consensual de la disciplina (incluso en algunos de estos “autores contemporáneos”) a tratar el problema de la pluralidad y la diversidad, disolviendo categorías totalizadoras de lo social.Creemos que es mucho más rico un debate sobre estas cuestiones a partir de nuevos útiles conceptuales, más que de ajustes de viejos paradigmas. En este sentido, nuestra segunda motivaciónes de orden conceptual. Nos encontramos en el contexto de un conjunto de materiales sociológicos que con enfoques diversos no alcanzan a tener sistematicidad (Nardacchione y Acevedo, 2014). En parte esto ocurre por su relativamente reciente aparición, pero fundamentalmente creemos que se debe a apropiaciones o lecturas heterogéneas de dichos materiales que, en muchos casos, tienden a “confundir” su base teórica-epistemológica, tanto a través de su inscripción dentro de una matriz no necesariamente coherente $\mathrm{s}^{\underline{5}}$, como a través de su confinamiento a una disciplina ámbito problemático específico ${ }^{6}$.En ese sentido, este dossier pretende colaborar a una clarificación de estos abordajes post-contemporáneos,aportandoasí a una sistematización que rinda justica a dichos debates y que sea comprendida como tal. Y en última instancia, que pueda ser enseñada de manera consistente.

Por otra parte,para organizar el dossierhemos procurado evitar las disociaciones tajantes entreteoría, metodología y práctica. Por lo tanto, además de presentar reflexiones y debates teóricometodológicas hemos puesto cierto énfasis en la consideración de sus apropiaciones en el marco de investigaciones empíricas.Esta lógica se refleja en la estructura del dossier, que incluye artículos originales, debates, notas de investigación y lecturas, además de una entrevista con Bernard Lahire a propósito de los temas que nos convocan.

En la sección “Artículos” contamos con tres ensayos teóricos que recorren parte de la obra de Bourdieu y la problematizan frente a distintos autores o cuestiones conceptuales. En el primer caso, 
Nardacchione y Tovillas centran su estudio sobre las obras de Bourdieu y Boltanski, como representantes (ambos) del estructuralismo genético que fundó el primero y, en el caso del segundo, como cofundador de la sociología pragmática francesa. En un sopesado análisis de rupturas y continuidades, se contrastan ambas obras frente a dos cuestiones cruciales de la sociología: el rol del investigador y el problema de la articulación entre subjetividad y objetividad. En el segundo artículo, Quéré realiza un estudio detallado sobre las posibles articulaciones entre la obra de Bourdieu y el pragmatismo norteamericano en relación con la cuestión del hábito. Allí, reconoce afinidades entre el habitusbourdiano y el habit en Dewey, pero también explora diferencias entre un enfoque de orden socio-disposicional y otro más bien ligado a la capacidad de acción. Por último, Benzecry analiza los diferentes usos de la noción dehabitus en la obra de Bourdieu, mostrando principalmente la manera en que el autor fue retomado en la sociologíanorteamericana. Desde allí se sumerge en ciertas aporías frente a las que se encuentra el uso extensivo del término, recuperando críticas estadounidenses y francesas a versionesen excesodisposicionalistas de la acción social. En suma, en esta sección atravesamos de manera crítica la obra de Bourdieu, tanto en Francia como en EE.UU., desde abordajesde tinte fundamentalmente pragmatista.

En la sección "Notas de investigación” se presentan reportes de investigaciones empíricas latinoamericanas, recientes o en curso, que han puesto el foco en las corrientes teóricas objeto del presente dossier (o en algunos de sus exponentes), o que se enmarcan en ellas, recuperan sus debates o se apropian y emplean algunas de sus categorías conceptuales centrales y/o sus propuestas metodológicas. Balerdiet alanalizan ciertos postulados habitualmente asociados con las corrientes pragmatistas, focalizándose en algunas obras de Callon, Hennion y Latour. Más específicamente, exploran las orientaciones metodológicas que se despliegan en relación con los principios de "seguir a los actores", de la "acción haciéndose" y de "simetría" en un corpus de investigaciones empíricas de los autores ya citados. PaschkesRoniset al ilustran un conjunto de investigaciones diversas, aunque atravesadas por un elemento común: el conocimiento y aprendizaje de los actores en contextos no institucionalizados, destacando la experimentación y la creatividad de los actores en espacios de acción novedosos. Bacal investiga el surgimiento de nuevos agentes artísticos en Brasil que consideran a las tecnologías digitales como elemento constitutivo de su creatividad y a los que define con la categoría de productores, reconstruyendo sus espacios de sociabilidad, así como sus procesos creativos y performances. Por último, Jiron y Lange presentan una investigación sobre movilidad urbana centrada en el caso del Transantiago (Chile). En su artículo, ponen en evidencia cómo los usuarios de este sistema de transporte, que se implementó sin haber considerado las prácticas de movilidad cotidiana de los habitantes de la ciudad, han reaprendido a viajar y han creado nuevas espacialidades, lo que demuestra los nexos que existen entre las prácticas sociales de movilidad, sus procesos de aprendizaje y la producción de saberes para comprender la ciudad.

La sección “Debates”abre con un texto deBoix ySemánen el que se señalan los importantes aportes de AntoineHennion para las ciencias sociales, cuyos esfuerzos, según los autores, "han intentado cuestionar los límites del construccionismo para dar lugar a una sociología de las irreversibilidades que integre en ensambles heterogéneos las relaciones entre lo humano y lo no humano”, abriendo laposibilidad de entender bajo una nueva luz "tanto los objetos de la cultura como el papel clave de las decisiones y estrategias metodológicas en las investigaciones que su programa propone”. A continuación, el mismo Hennion presentauna mirada retrospectiva y reflexiva de su propia 
trayectoria en el Centre de Sociologie de l'Innovation (CSI)a partir de la discusión de las convergencias y divergencias en el trabajo de campo realizado en dos líneas de trabajo: ciencia y tecnología, por un lago, y cultura, por el otro. Luego analiza la emergencia de los enfoques pragmatistas en Francia y cómo estos informaronsus propias investigaciones.Para finalizar,señala la importancia de que la sociología se torne más sensible en relación con los objetos de los que se ocupa.

Para la sección “Entrevista”convocamos al prolífico sociólogo francés B. Lahire, que detalla su crítica "pluralista" a la obra de Bourdieu alavez que plantea serios cuestionamientos al enfoque pragmatista. En ese sentido, su posturamuestra diferencias con respecto a las versiones más holistas de la sociología bourdiana, pero llegado el momento, toma aún más distancia del enfoque "situado", proveniente del pragmatismo,al que califica como insuficientepor no considerar la influencia de factores no evidentes en el contexto de la acción (trayectorias, historias, etc.).

Finalmente, en la sección "Lecturas", Lio y Urtasun reseñan el libro Enigmas y complots. Una investigación sobre las investigaciones, de LucBoltanski, recientemente publicado en español por Fondo de Cultura Económica. Cuestas y Hang, por su parte, presentan una recensión del libro de TiaDeNora, After Adorno. RethinkingMusicSociology (Cambridge UniversityPress), que no cuenta con traducción española. Y PaschkesRonis cierra la sección con una reseña del libro Un relato de la Globalización desde el Chaco, de Mario Blaser, publicado por la Editorial Universidad del Cauca.

\section{Notas}

1 Dicho "consenso de mínima” podría adjudicarse a que cada uno de estos tres autores representa, en algún sentido, una primera huella teórica y epistemológica, que posteriormente va a ir sofisticándose con el desarrollo de la disciplina. Nos referimos a la tradición crítica-estructural marxista, la tradición positiva durkheimiana y la tradición comprensiva weberiana.

\section{$\underline{2}$ http://www.memoria.fahce.unlp.edu.ar/programas/pp.527/pp.527.pdf}

$\underline{3}$ Es ya un lugar común dentro de la historia de la sociología la relación de asimetría que a mediados del siglo XX existía entre el paradigma estructural-funcionalista parsoniano y los estudios así llamados interaccionistas o etnometodológicos. Estos fueron incluso frecuentemente “degradados” a tópicos micro-sociológicos o a meros puntos de vista metodológicos.

4 Entre sus padres fundadores hacemos referencia, en principio, a las públicas controversias entre Peirce y James, y aún a las persistentes diferencias entre James y Dewey. Luego, sus ciclos de aparición y reaparición, desde supuestos no siempre coherentes, van a manifestarse en diversas esferas del conocimiento (filosofía de la ciencia, lógica, ética). De la misma manera, dentro de la sociología como disciplina, dichas diferencias van a manifestarse dentro del recorrido de la Escuela de Chicago, la que de alguna manera va a representar dicha tradición. A menudo es difícil encontrar un enfoque sistemático entre lo que se dio en llamar laprimera escuela de Chicago (Thomas, Park, Burgess) y la segunda escuela de Chicago (Blumer). El mismo tipo de semejanzas y diferencias se encuentra en relación con la sociología goffmaniana y la sociología de la desviación de Becker. 
$\underline{5}$ Así, por ejemplo, se enseña la obra de Boltanski(1971; c/ Bourdieu, Castel y Chamborendon, 2003) sin distinguir su ruptura con el paradigma bourdiano, o se asocia la teoría del actor-red de Latour(2008) con la propuesta de sociedad post-industrial en red de Castells.

6 Por caso, la obra de Latour(1995) tiende a ser estudiada en América Latina como un aporte específico a los estudios sociales de la ciencia, así como a menudo se relaciona aBoltanski (2002)con estudios organizacionales de un capitalismo managerial.

\section{Bibliografía}

Aron, R. (1967). Les Étapes de la pensée sociologique. Paris: Gallimard.

Baranger, D. (2011). Antropología social y sociología argentinas: identidades disciplinares en cuatro congresos. Revista Latinoamericana de Metodología de las Ciencias Sociales, 1 (2), pp. 23-59.

Barthe, Y.et al. (2016). Sociología pragmática: guia do usuario.Sociologías, 18 (41), p. 84-129.

Bialakowsky, A. (2010). Comunidad y sentido en la teoría sociológica contemporánea: las propuestas de A. Giddens y J. Habermas. Papeles del CEIC, 53,

Boltanski, L. (1971). Les usages sociaux du corps. Annales. Économies, sociétés, civilisations, 26, p. 205-233.

Boltanski, L. y Chiapello, E. (2002).El nuevo espíritu del capitalismo. Madrid: Akal.

Bourdieu, P. yPasseron, J. C. (1970).La Réproduction, éléments pour une théorie du système d'enseignement. Paris : Minuit.

Bourdieu, P. (1980).Le sens pratique. Paris : Minuit.

Bourdieu,P. (1988) La Distinción, criterios y bases sociales del gusto. Madrid: Taurus.

Bourdieu, P.,Castel, R., Boltanski, L. y Chamboredon, J. C. (2003).Un arte medio. Ensayo sobre los usos sociales de la fotografía. Barcelona: Gustavo Gili.

Breviglieri, M. y Stavo-Debauge, J. (1999). Le geste pragmatique de la sociologie française. Autour des travaux de Luc Boltanski et Laurent Thévenot.Antropolítica, 7, pp. 7-22.

Commeti, J. P. (2010) Qu'est-ce que le pragmatisme? Paris : Gallimard.

Dubet, F. (1996). ¿¿caso de la idea de sociedad? Revista de Sociología (Chile), 10, pp. 7-23

Gane, N. (2005). Crítica a la teoría social clásica. Trayectorias, VII (19), pp. 7-18.

Hernández Romero, Y. y Galindo Sosa, R. V. (2009). La contemporaneidad del pensamiento de Durkheim, Weber y Marx. Espacios Públicos, 12 (24), pp. 189-201.

Lamo de Espinosa, E. (2001). La sociología del siglo XX. Revista Española de Investigaciones Sociológicas, 96, pp. 21-49

Latour, B. y Woolgar, S. (1995).La vida en el laboratorio. La construcción de los hechos científicos. Buenos Aires : Alianza Editorial. 
Nardacchione, G. y Acevedo, M.(2014.) Las sociologías pragmático-pragmatistas puestas a prueba en América Latina.Revista Argentina de Sociología, 17-18, pp. 86-118.

Nisbet, R. (1969). La formación del pensamiento sociológico. Buenos Aires: Amorrortu.

Pérez de Tudela, J. (2008) El Pragmatismo americano. Madrid: Síntesis.

Piovani, J. I. (2015). Methodological interests and the teaching of research methods in Latin America.Sociologies in Dialogue. Journal of the Brazilian Sociological Society, 1 (1), pp. 106-118

Ritzer, G. (1993). Teoría social clásica. Madrid: MacGraw-Hill.

Zabludovsky, G. (1991). Clásicos y contemporáneos en la teoría sociológica. Entrevista con Jeffrey Alexander. Acta Sociológica, IV (2-3), pp. 17-34 